\title{
The correlation between urinary 5-hydroxyindoleacetic acid and sperm quality in infertile men and rotating shift workers
}

\author{
Águeda Ortiz ${ }^{1 \dagger}$, Javier Espino ${ }^{2^{*}}$, Ignacio Bejarano², Graciela M Lozano', Fabián Monllor ${ }^{1}$, Juan F García ${ }^{1}$, \\ José A Pariente ${ }^{2}$, Ana B Rodríguez²
}

\begin{abstract}
Background: Serotonin is a neurotransmitter that modulates a wide range of neuroendocrine functions. However, excessive circulating serotonin levels may induce harmful effects in the male reproductive system. The objective of this study was to evaluate whether the levels of urinary 5-hydroxyindoleacetic acid (5-HIIA), a major serotonin metabolite, correlate with different classical seminal parameters.

Methods: Human ejaculates were obtained from 40 men attending infertility counselling and rotating shift workers by masturbation after 4-5 days of abstinence. Urinary 5- HIIA concentration was quantified by using a commercial ELISA kit. Forward motility was assessed by a computer-aided semen analysis (CASA) system. Sperm concentration was determined using the haemocytometer method. Sperm morphology was evaluated after Diff-Quik staining, while sperm vitality was estimated after Eosin-Nigrosin vital staining.

Results: Our results show that urinary 5-HIIA levels obtained from a set of 20 volunteers negatively correlated with sperm concentration, forward motility, morphology normal range and sperm vitality. On the other hand, we checked the relationship between male infertility and urinary 5-HIIA levels in 20 night shift workers. Thus, urinary 5-HIIA levels obtained from 10 recently-proven fathers were significantly lower than those found in 10 infertile males. Additionally, samples from recent fathers exhibited higher sperm concentration, as well as better forward motility and normal morphology rate.
\end{abstract}

Conclusions: In the light of our findings, we concluded that high serotonin levels, indirectly measured as urinary 5-HIIA levels, appear to play a role as an infertility determinant in male subjects.

\section{Background}

Standard parameters evaluated, i.e. sperm morphology and motility, have shown different associations with male fertility. Particularly, Zhang et al. [1] demonstrated significant correlations among in vitro semen tests (total sperm concentration and motile sperm), zona pellucidabinding assay, and in vitro blastocyst production. On the other hand, the integrity of the plasma membrane reflects sperm viability and several process related to sperm physiology, such as capacitation, acrosome reaction, and binding of spermatozoa to the oocyte surface,

\footnotetext{
* Correspondence: jespino@unex.es

† Contributed equally

${ }^{2}$ Department of Physiology, Neuroimmunophysiology and Chrononutrition Research Group, Faculty of Science, University of Extremadura, Badajoz, Spain Full list of author information is available at the end of the article
}

require a biochemically active membrane $[2,3]$. Considering that capacitated and/or acrosome- reacted spermatozoa have a limited life span [4], this would result in impaired fertility.

The major function of the circadian system is the internal cycling of physiologic and metabolic events. Circadian rhythms are synchronized to the 24-h day, mostly by light-dark cycles, partially by other environmental and social time cues $[5,6]$. The circadian rhythm can get desynchronized in rotating shift workers since their night activity is out of phase, and such desynchronization may contribute to important health problems. For instance, the effects of rotating and night shift work on female fertility have been reported [7]. Additionally,

\section{Biomed Central}

(c) 2010 Ortiz et al; licensee BioMed Central Ltd. This is an Open Access article distributed under the terms of the Creative Commons Attribution License (http://creativecommons.org/licenses/by/2.0), which permits unrestricted use, distribution, and reproduction in any medium, provided the original work is properly cited. 
undergoing night shift work may alter secretion circadian rhythms, including serotonin secretion [8].

Serotonin is a neurotransmitter involved in a wide range of behavioural and physiological processes. In fact, serotonergic neurons play an important role modulating neuroendocrine functions such as food intake, sleep, mood and sexual behaviour [9]. In the male reproductive system, serotonin might affect directly sperm maturation since the existence of a local serotonergic system in the rat caput epididymis have been described [10]. However, relatively recent studies have reported that hyperserotoninaemia may relate to certain kinds of male infertility $[11,12]$. In this regard, several studies have pointed out that selective serotonin reuptake inhibitors, which are commonly prescribed as antidepressants, can impair semen quality and damage sperm DNA integrity $[13,14]$. Therefore, up to now, the relationship between serotonin and male fertility still remains unclear. To this end, we aimed to evaluate whether the levels of urinary 5-hydroxyindoleacetic acid (5-HIIA), a major serotonin metabolite, correlate with different seminal parameters, including sperm concentration, motility and morphology, in a set of individuals attending infertility counselling. On the other hand, we checked the relationship between male infertility and urinary 5-HIIA levels in night shift workers by comparing 5-HIIA levels and seminal parameters between a group of recent fathers and another of infertile men, all of them undergoing night shift work.

\section{Methods}

\section{Semen collection and preparation}

Human semen was obtained from 40 men (20-40 year -old), as approved by the institutional review board of the University of Extremadura and the ethics committee of Infantile Hospital (Badajoz, Spain), as well as in accordance with the Declaration of Helsinki. Each subject was ascertained to be in good health by means of their medical histories and a clinical examination including routine laboratory test and screening. The subjects were all non smokers, were not using any medication and abstained from alcohol. Informed consent was obtained from all the participants. Samples were collected by masturbation after 4-5 days of sexual abstinence and allowed to liquefy $\left(30\right.$ minutes, $37^{\circ} \mathrm{C}$ ) before processing. All samples were collected at the same time of the year, thereby avoiding the seasonal and photoperiodic variations of sperm sample [15].

Routine seminal parameters were evaluated according to the World Health Organization criteria [16]. Thus, forward motility (grade $a+b$ sperm motility) was assessed by a computer-aided semen analysis (CASA) system (Sperm Class Analyser, Microptic S.L., Spain). Sperm concentration, expressed as $10^{6}$ cells $/ \mathrm{mL}$, was determined using the haemocytometer method on two separate preparations of the semen sample. Sperm morphology, measured as percentage of normal cells, was evaluated after Diff-Quik, while sperm vitality, assessed as percentage of viable cells, was estimated after EosinNigrosin vital staining, which has been developed to evaluate sperm membrane integrity and let us distinguish intact, live spermatozoa from those that have lost their sperm membrane integrity (dead spermatozoa).

\section{Experimental design}

The design of this study consisted of two parts. First of all, an in vivo study examined the link between urinary levels of 5-HIIA and sperm quality. Thus, we evaluated whether 5-HIIA levels measured in 20 volunteers correlated with different seminal parameters. These subjects were a mixture of infertile and fertile men. Normozoospermia was indicated by sperm concentration $\geq 20 \times 10^{6}$ cells/mL, progressive motility (grade a $+\mathrm{b}$ sperm motility) $\geq 50 \%$, and normal sperm morphology $\geq 14 \%$.

Secondly, we also examined whether serotonin, indirectly measured as urinary 5-HIIA, may influence sperm quality in 20 night shift workers. To this end, we compared the different seminal parameters between 10 recently-proven fathers, named as fertile group, and 10 infertile males, named as infertile group.

\section{Measurement of 5-hydroxyindoleacetic acid in urine}

For urinary 5- HIIA determination, urines were collected at 19:00 h. The samples were stored at $-20^{\circ} \mathrm{C}$ until biochemical assay. Urinary 5 -HIIA, which is a major serotonin metabolite, was quantified by using a commercial ELISA kit (IBL, Hamburg, Germany) according to the manufacturer's instructions. To adjust for variation in the dilution of urine, 5-HIIA concentrations were expressed as urinary 5- HIIA/urine creatinine. Creatinine concentration was determined by means of the Jaffe test, as described elsewhere [17].

\section{Statistical analysis}

Pearson's correlation by multiple regression of different seminal parameters with the 5-HIIA levels were tested. Student's t-test was used at the significance level $\alpha=$ 0.05 . Data displayed in histograms are expressed as mean \pm SEM. All analyses were performed using GraphPad Prism 5 (GraphPad Software Inc., San Diego, CA, USA).

\section{Results and Discussion}

Figure 1 shows the correlations between urinary 5-HIIA levels, obtained from 20 volunteers, and different seminal parameters analysed in fresh ejaculates. Curiously, urinary 5-HIIA levels negatively correlate with sperm concentration (statistically significant), forward motility (statistically significant), morphology normal range (statistically significant) and sperm vitality (statistically 


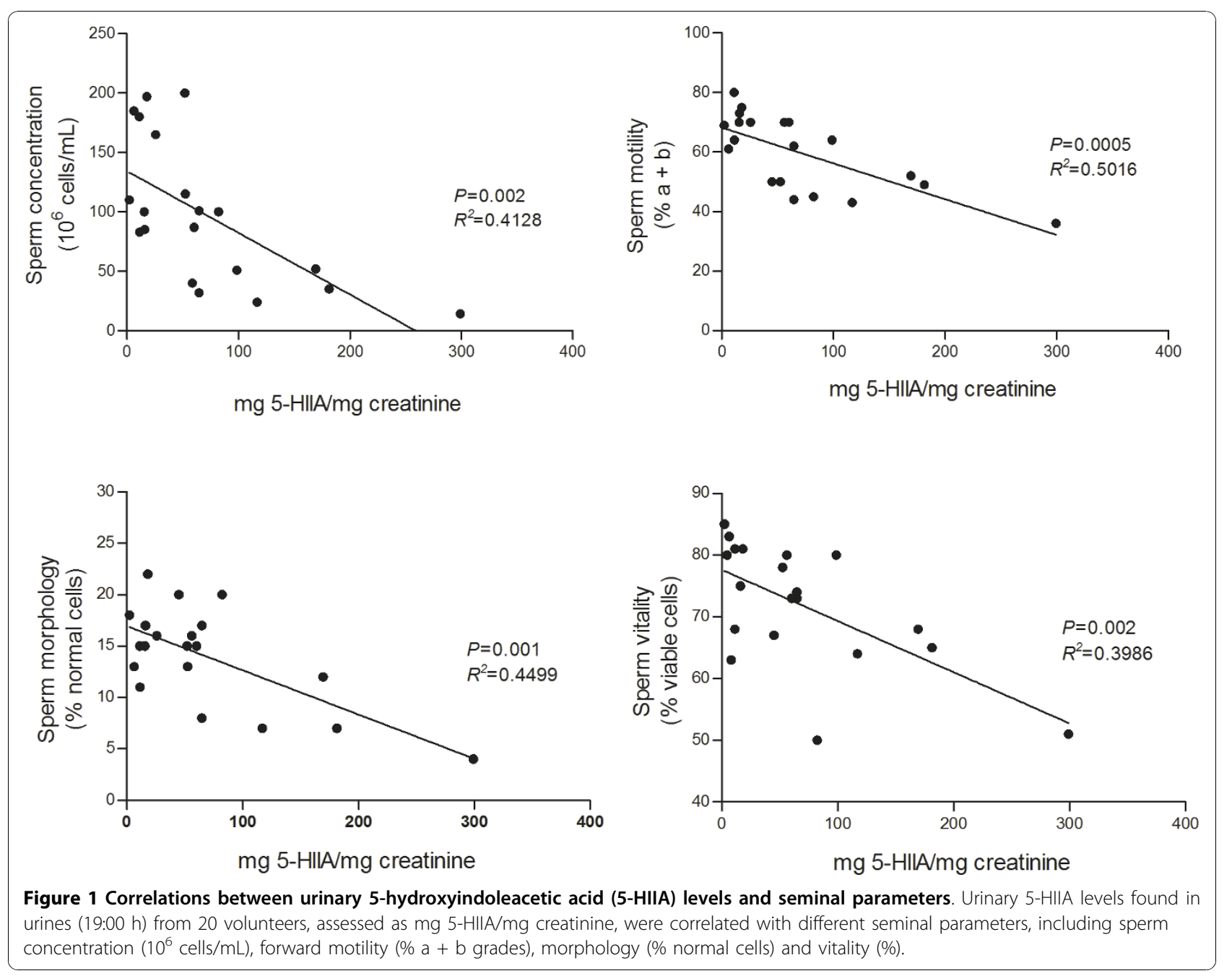

significant). Taken together, these findings point out that elevated urinary 5-HIIA levels may worsen sperm quality, contrary to our expectations.

To verify whether urinary 5-HIIA levels may influence sperm quality, we compared urinary 5-HIIA levels found in urines $(19: 00 \mathrm{~h})$ from recently-proven fathers $(\mathrm{n}=$ $10)$, designed as fertile, with those obtained from infertile males $(n=10)$. All volunteers were working the night shift work, which is one of the facts that may alter serotonin circadian secretion [4]. Interestingly, urinary 5-HIIA levels measured in recently-proven fathers are lower (Figure 2, statistically significant) than those found in infertile males. In addition, we further investigated the relationship between serotonin and sperm quality by comparing different seminal parameters in both fertile and infertile night shift workers (Table 1). Our results display that samples from recently- proven fathers exhibit a higher sperm concentration (statistically significant), an increased forward motility (statistically significant), as well as an elevated normal morphology rate (statistically significant), compared with those values obtained in samples from infertile males. No differences were found with regards to sperm vitality (unpublished data). Therefore, the elevated serotonin endogenous levels, indirectly assessed as urinary 5-HIIA, may impair sperm quality, thus inducing male infertility in night shift workers.

Male reproduction is likely controlled, at least partly, by circadian system. In fact, melatonin, an indoleamine whose production is subject to circadian rhythms with high levels at night and low by day [18], is able to modulate the reproductive physiology due to the melatonin regulator effect on the hypothalamus-pituitary-testicular axis [19]. Likewise, it has been reported that melatonin positively affects sperm physiology and may strongly protect spermatozoa from the oxidant environment produced by excessive round cells in seminal fluid $[20,21]$.

Over the past few years, studies have proven that serotonin, another component of the circadian system, is necessary for the development of normal spermatogenesis in 


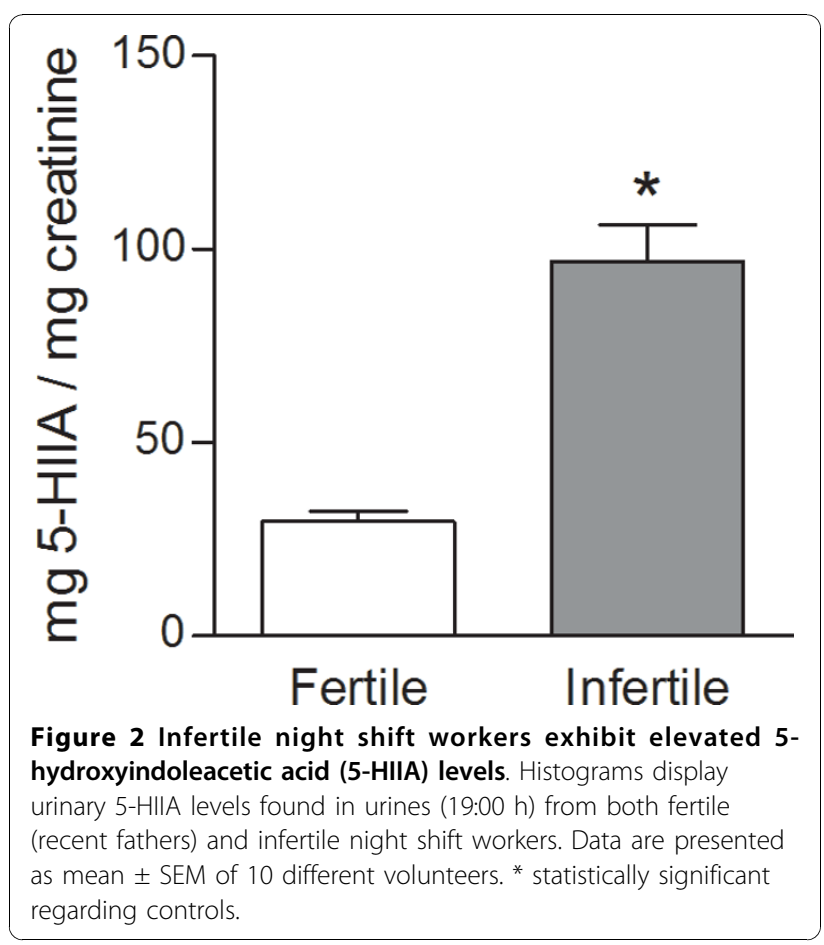

rats [22] owing to the existence of a local serotonergic system in the rat epididymis [10]. Nevertheless, as abovementioned, high levels of serotonin appear to play a role as an infertility determinant in male subjects suffering from varicocele [12], or even azoospermy [23], i.e. an excess of circulating serotonin may induce harmful effects on male reproductive system. In fact, long-term treatments with antidepressants which enhance the availability of endogenous serotonin, such as sertraline or citalopram, can impair semen quality $[13,14]$. Despite both rats and human beings are mammals, we cannot assume that serotonin plays the same role on male reproductive system in both species because serotonin, which is also involved in sleep control [9], differently affects sleep/wake patterns in rodents and humans. In this sense, our findings shed some light on the relationship between serotonin and male infertility since we showed that the levels of urinary 5HIIA negatively correlate with semen quality, as assessed by sperm concentration, motility or morphology.

Table 1 Seminal quality in night shift workers

\begin{tabular}{ccc}
\hline Seminal Parameters & $\begin{array}{c}\text { Fertile group } \\
(\mathbf{n}=\mathbf{1 0})\end{array}$ & $\begin{array}{c}\text { Infertile group } \\
(\mathbf{n}=\mathbf{1 0})\end{array}$ \\
\hline $\begin{array}{c}\text { Sperm concentration } \\
(\mathbf{1 0 6} \text { cells/mL) }\end{array}$ & $148.0 \pm 6.95$ & $112.8 \pm 3.35^{*}$ \\
$\begin{array}{c}\text { Forward motility } \\
\text { (\% a + b grades) } \\
\quad \text { Morphology } \\
\text { (\% normal cells) }\end{array}$ & $54.50 \pm 1.04$ & $39.67 \pm 1.85^{*}$ \\
\hline
\end{tabular}

Data are presented as mean \pm SEM of the number of volunteers.

*statistically significant regarding fertile group.

\section{Conclusions}

To sum up, elevated serotonin endogenous levels, indirectly assessed as urinary 5-HIIA, likely induce poor sperm quality that, consequently, leads to subfertility problems. In the light of these results, it is feasible to presume that a high dose of serotonin locally applied to the testicular surrounding area would be used as a reversible, cost-effective male contraceptive method. However, further studies are required to clarify molecular mechanisms through which serotonin modulates male reproductive system and to test whether serotonin supplementation has any noticeable side effect, such as sexual dysfunctions or mood changes.

\section{List of abbreviations}

CASA: computer-aided semen analysis; 5-HIIA: 5-hydroxyindoleacetic acid.

\section{Acknowledgements}

This work was supported by Merck, S.L. J. Espino and I. Bejarano are supported by Merck, S.L. and Junta de Extremadura (PRE06070) fellowships, respectively.

\section{Author details}

${ }^{1}$ Extremadura Centre for Human Assisted Reproduction, Badajoz, Spain. ${ }^{2}$ Department of Physiology, Neuroimmunophysiology and Chrononutrition Research Group, Faculty of Science, University of Extremadura, Badajoz, Spain.

\section{Authors' contributions}

$\mathrm{AO}$ and JE carried out the experiments and drafted the manuscript. IB performed the statistical analysis and helped in drafting the manuscript. GML, FM and JFG collected sperm samples and performed the analysis of sperm parameters. JAP and ABR designed and conceived the study, interpreted the data and discussed the results. All authors read and approved the final manuscript.

\section{Competing interests}

The authors declare that they have no competing interests.

Received: 4 October 2010 Accepted: 8 November 2010 Published: 8 November 2010

\section{References}

1. Zhang BR, Larsson B, Lundeheim N, Haard MG, Rodriguez-Martínez H: Prediction of bull fertility by combined in vitro assessments of frozenthawed semen from young dairy bulls entering an Al-program. Int J Androl 1999, 22:253-260.

2. Jeyendran RS, Van Der ven HH, Perez-Pelaes M, Crabo BG, Zaneveld LJD: Development of an assay to assess the functional integrity of the human sperm membrane and its relationship to other semen characteristics. J Reprod Fertil 1984, 70:219-228.

3. Thundathil J, Gil J, Januskauskas A, Larsson B, Soderquist L, Mapletoft R, Rodriguez-Martinez R: Relationship between the proportion of capacitated spermatozoa present in frozen-thawed bull semen and fertility with artificial insemination. Int J Androl 1999, 22:366-373.

4. Yanagimachi R: Mammalian fertilization. In The physiology of reproduction Edited by: Knobil E, Neill JD. New York: Raven Press; 1994:189-315.

5. Oster H: The genetic basis of circadian behavior. Genes Brain Behav 2006, 5:73-79.

6. Berger JA: A two-clock model of circadian timing in the immune system of mammals. Pathol Biol 2008, 56:286-291.

7. Ahlborg G, Axelsson G, Bodin L: Shift work, nitrous oxide exposure and subfertility among Swedish midwives. Int J Epidemiol 1996, 25:783-790.

8. Sookoian S, Gemma C, Fernández-Guianotti T, Burgueño A, Alvarez A, González CD, Pirola CJ: Serotonin and serotonin transporter gene variant in rotating shift workers. Sleep 2007, 30:1049-1053. 
9. Murphy DL, Fox MA, Timpano KR, Moya PR, Ren-Patterson R, Andrews AM, Holmes A, Lesch KP, Wendland JR: How the serotonin story is being rewritten by new gene-based discoveries principally related to SLC6A4, the serotonin transporter gene, which functions to influence all cellular serotonin systems. Neuropharmacology 2008, 55:932-960.

10. Jiménez-Trejo F, Tapia-Rodríguez M, Queiroz DB, Padilla P, Avellar MC, Manzano PR, Manjarrez-Gutiérrez G, Gutiérrez-Ospina G: Serotonin concentration, synthesis, cell origin, and targets in the rat caput epididymis during sexual maturation and variations associated with adult mating status: morphological and biochemical studies. J Androl 2007, 28:136-149.

11. Gonzales GF, Garcia-Hjarles M, Velasquez G: Hyperprolactinaemia and hyperserotoninaemia: their relationship to seminal quality. Andrologia 1992, 24:95-100.

12. Gonzales GF, Garcia-Hjarles MA: Blood/seminal serotonin levels in infertile men with varicocele. Arch Androl 1990, 24:193-199.

13. Tanrikut C, Schlegel PN: Antidepressant-associated changes in semen parameters. Urology 2007, 69:185.e5-185.e7.

14. Safarinejad MR: Sperm DNA damage and semen quality impairment after treatment with selective serotonin reuptake inhibitors detected using semen analysis and sperm chromatin structure assay. J Urol 2008, 180:2124-2128.

15. Krause A, Krause W: Seasonal variations in human seminal parameters. Eur J Obstet Gynecol Reprod Biol 2002, 101:175-178.

16. World Health Organization: WHO Laboratory Manual for the Examination of Human Semen and Semen-Cervical Mucus Interaction. 4 edition. Cambridge University Press; Cambridge; 1999.

17. Klante G, Brinschwitz T, Secci K, Wollnik F, Steinlechner S: Creatinine is an appropriate reference for urinary sulphatoxymelatonin of laboratory animals and humans. J Pineal Res 1997, 23:191-197.

18. Sánchez $S$, Sánchez $C L$, Paredes SD, Rodríguez $A B$, Barriga C: The effect of tryptophan administration on the circadian rhythms of melatonin in plasma and the pineal gland of rats. J Appl Biomed 2008, 6:177-186.

19. Casao A, Cebrián I, Asumpção ME, Pérez-Pé R, Abecia JA, Forcada F, Cebrián-Pérez JA, Muiño-Blanco T: Seasonal variations of melatonin in ram seminal plasma are correlated to those of testosterone and antioxidant enzymes. Reprod Biol Endocrinol 2010, 8:59.

20. Du Plessis SS, Hagenaar K, Lampiao F: The in vitro effects of melatonin on human sperm function and its scavenging activities on NO and ROS. Andrologia 2010, 42:112-116.

21. Ortiz A, Espino J, Bejarano I, Lozano GM, Monllor F, García JF, Pariente JA, Rodríguez AB: High endogenous melatonin concentrations enhance sperm quality and short-term in vitro exposure to melatonin improves aspects of sperm motility. J Pineal Res 2010.

22. Aragón MA, Ayala ME, Marín $M$, Avilés A, Damián-Matsumura $P$, Domínguez R: Serotoninergic system blockage in the prepubertal rat inhibits spermatogenesis development. Reproduction 2005, 129:717-727.

23. Segal S, Sadovsky E, Palti Z, Pfeifer Y, Polishuk WZ: Serotonin and 5hydroxyindoleacetic acid in fertile and subfertile men. Fertil Steril 1975, 26:314-316.

doi:10.1186/1477-7827-8-138

Cite this article as: Ortiz et al:: The correlation between urinary 5hydroxyindoleacetic acid and sperm quality in infertile men and rotating shift workers. Reproductive Biology and Endocrinology 2010 8:138.

\section{Submit your next manuscript to BioMed Central and take full advantage of:}

- Convenient online submission

- Thorough peer review

- No space constraints or color figure charges

- Immediate publication on acceptance

- Inclusion in PubMed, CAS, Scopus and Google Scholar

- Research which is freely available for redistribution

Submit your manuscript at www.biomedcentral.com/submit 